Please do not remove this page

RMIT

UNIVERSITY

\title{
Development of a new biochemical test to diagnose and monitor neuroblastoma in Vietnam: Homovanillic and vanillylmandelic acid by gas chromatography-mass spectrometry
}

Tran, Mai; Baglin, James; Tran, Trang; Hoang, Kien; Phung, Lan; Read, Ann; Greaves, Ronda https://researchrepository.rmit.edu.au/esploro/outputs/9921859399901341/filesAndLinks?institution=61RMIT_INST\&index=null

Tran, M., Baglin, J., Tran, T., Hoang, K., Phung, L., Read, A., \& Greaves, R. (2014). Development of a new biochemical test to diagnose and monitor neuroblastoma in Vietnam: Homovanillic and vanillylmandelic acid by gas chromatography-mass spectrometry. Clinical Biochemistry, 47(3), 206-215.

https://doi.org/10.1016/j.clinbiochem.2013.11.016

Document Version: Submitted Version

Published Version: https://doi.org/10.1016/j.clinbiochem.2013.11.016

Repository homepage: https://researchrepository.rmit.edu.au

(c) 2013 The Canadian Society of Clinical Chemists. Published by Elsevier Inc. All rights reserved

Downloaded On 2023/04/27 00:59:18 +1000 
Thank you for downloading this document from the RMIT Research Repository.

The RMIT Research Repository is an open access database showcasing the research outputs of RMIT University researchers.

RMIT Research Repository: http://researchbank.rmit.edu.aul

\section{Citation:}

Chi, M, Baglin, J, Tran, T, Hoang, K, Phung, L, Read, A and Greaves, R 2013, 'Development of a new biochemical test to diagnose and monitor neuroblastoma in Vietnam: Homovanillic and vanillylmandelic acid by gas chromatography-mass spectrometry', Clinical Biochemistry, vol. 47, no. 3, pp. 206-215.

See this record in the RMIT Research Repository at:

https://researchbank.rmit.edu.au/view/rmit:23623

Version: Submitted Version

\section{Copyright Statement:}

(c) 2013 The Canadian Society of Clinical Chemists. Published by Elsevier Inc. All rights reserved

Link to Published Version:

https://researchbank.rmit.edu.au/view/rmit:23623 
Biochemical diagnosis and monitoring of Neuroblastoma in Vietnam: Development of homovanillic and vanillylmandelic acid analysis by gas chromatography - mass spectrometry and establishment of reference intervals Highlights

- A cost effective and robust testing method for simultaneous quantitation of urinary HVA and VMA by GCMS was developed.

- Age - related reference intervals for random urine HVA and VMA samples were established for Vietnamese children.

- The intervals declined progressively with increasing age for each analyte.

- The appropriate assignment of the upper reference interval for these biomarkers will ensure maximum specificity for neuroblastoma diagnosis and treatment monitoring. 
Original Research Communication - Analytical

Biochemical diagnosis and monitoring of Neuroblastoma in Vietnam: Development of homovanillic and vanillylmandelic acid analysis by gas chromatography - mass spectrometry and establishment of reference intervals.

Short title: Vietnam GCMS HVA - VMA reference interval study.

Authors: Mai T.C. Tran ${ }^{1,2}$, James Baglin ${ }^{3}$, Trang T.T. Tran ${ }^{1}$, Kien H. Trung ${ }^{1}$, Lan T. Phung ${ }^{4}$, Ann Read ${ }^{5}$, Ronda F. Greaves ${ }^{6,7}$

${ }^{1}$ Clinical Biochemistry Laboratory, National Hospital of Pediatrics, Ha Noi, Viet Nam

${ }^{2}$ Hanoi Medical University, Ha Noi, Viet Nam

${ }^{3}$ School of Mathematical and Geospatial Sciences, RMIT University, Victoria, Australia

${ }^{4}$ Oncology Department, National Hospital of Pediatrics, Ha Noi, Viet Nam

${ }^{5}$ Eastern Health Pathology, Box Hill Hospital, Victoria, Australia

${ }^{6}$ School of Medical Sciences, RMIT University, Victoria, Australia

${ }^{7}$ Centre for Hormone Research, Murdoch Children's Research Institute, Victoria, Australia.

Corresponding author: Dr Ronda Greaves, School of Medical Sciences, RMIT University, PO Box 71, Bundoora, Victoria, Australia 3083.

Tel: +61 (0)39925 7080; email: ronda.greaves@rmit.edu.au

Key words: reference intervals, HVA, VMA, neuroblastoma, GC-MS, random urine, mass spectrometry

\section{Declarations}

Competing interests: None 
Funding: Asian-Pacific Federation for Clinical Biochemistry and Laboratory Medicine (APFCB).

Ethical Approval: NHP356/HDKH

Guarantor: Ronda Greaves

Contributorship: All authors contributed equally to the development and analysis of this study. Drs Tran and Greaves jointly developed the project plan which was reviewed by $\mathrm{Dr}$ Read. $\mathrm{Dr}$ Tran, $\mathrm{Dr}$ Tran and Mr Hoang conducted the experiments in the Clinical Biochemistry Laboratory, National Hospital of Paediatrics Vietnam. Dr Phung selected the confirmed neuroblastoma patients for urine collection. Dr Greaves reviewed the method validation throughout the project during onsite visits and via performance in the RCPA Quality Assurance Program for urine chemistry. Dr Baglin performed the statistical analysis. $\mathrm{Dr}$ Tran wrote the first draft of this manuscript and all authors reviewed, edited and approved the final manuscript. 
Abstract

Objectives: The aim of this study was to develop an accurate robust testing method to simultaneously measure urine levels of HVA and VMA using gas chromatography mass spectrometry (GCMS) and to establish age-specific reference intervals of HVA and VMA in random urines for Vietnamese children.

Design and Methods: The assay for urinary HVA and VMA was developed and incorporated the internal standard 3-phenyl butyric acid, liquid-liquid extraction with ethyl acetate, derivatization with BSTFA followed by gas chromatographic separations with mass spectrometric detection in single ion monitoring mode.

Reference intervals were developed from random urine samples collected from 634 disease free Vietnamese children and compared to 50 known neuroblastoma patient samples. Results were reported relative to creatinine concentration. Age related $95 \%$ reference intervals for urinary HVA and VMA were estimated from sample quantiles.

Results: Method validation studies were performed prior to the establishment of the Vietnamese age related reference intervals for urinary HVA and VMA per mmol creatinine. HVA for children $<6$ months $(n=91)$ was $6.2-36.1 \mu \mathrm{mol} / \mathrm{mmol} ; 6$ months to $<1$ year $(n=141)$ was 3.7 to $25.9 \mu \mathrm{mol} / \mathrm{mmol} ; 1$ to 5 years $(n=139)$ was 3.5 to $16.5 \mu \mathrm{mol} / \mathrm{mmol} ; 6$ to 10 years $(\mathrm{n}=136)$ was $2.7-8.3 \mu \mathrm{mol} / \mathrm{mmol}$; and 11 to 15 years $(n=127)$ was $1.3-8.9 \mu \mathrm{mol} / \mathrm{mmol}$. VMA for children $<6$ months was 2.0 $11.9 \mu \mathrm{mol} / \mathrm{mmol}$; 6 months to $<1$ year was $1.7-9.1 \mu \mathrm{mol} / \mathrm{mmol} ; 1$ to 5 years was 2.1-7.4 $\mu \mathrm{mol} / \mathrm{mmol} ; 6$ to 10 years was $1.7-4.4 \mu \mathrm{mol} / \mathrm{mmol}$; and 11 to 15 years was $0-6.0 \mu \mathrm{mol} / \mathrm{mmol}$.

Conclusions: A robust testing method for simultaneous quantitation of urinary HVA and VMA by GCMS was developed. This method is accurate, precise and fit for its clinical purpose and suitable for developing countries. Age - related reference intervals of random urinary HVA and VMA were established for Vietnamese children and the intervals declined progressively with increasing age for each analyte. 


\section{INTRODUCTION}

Neuroblastoma, a neuroblastic tumor derived from primordial neural crest, is the most common extracranial solid tumor in childhood, and the most frequent diagnosed in infancy. If detected early enough, neuroblastoma is curable. Biochemically early diagnosis of the tumor can be done by determination of various biomarkers excreted continuously in elevated amounts in the urine of infants [1].

Neuroblastoma is characterized biochemically by the elevation of the urine catecholamine dopamine and catecholamine metabolites, particularly homovanillic (HVA) and vanillylmandelic acid (VMA). In over $92 \%$ of cases of neuroblastoma the presence of the tumor is accompanied by an increase in urinary HVA and / or VMA excretion.[2] Therefore, the measurement of these biomarkers is important in the diagnosis and monitoring of patients with neuroblastoma.

These biomarkers are routinely measured in developed countries by high performance liquid chromatography (HPLC) with electrochemical detection (ECD) and more recently with mass spectrometry (MS) detection. Such instrumentation is not routinely used in the Vietnamese hospital setting. In Vietnam, previously these analytes could not be measured and instead VMA by colorimetric analysis was performed. However, gas chromatography coupled mass spectrometry (GCMS) detection is newly available (since 2010) and we proposed to develop a method based on a traditional GCMS organic acids method [3].

The aim of this study was to develop a robust testing method to simultaneously measure urine levels of HVA and VMA using the GCMS at the National Hospital of Pediatrics (NHP) and to establish age-specific reference intervals of HVA and VMA in random urines for Vietnamese children. 


\section{MATERIALS AND METHOD}

\section{Sample collection}

Random urine samples were collected from 634 disease free children in preschools and schools around Hanoi Vietnam. These included 91 children less than 6 months, 141 from 6 months to less than 1 year, 139 from 1 to 5 years and 136 from 6 to 10 years, and 127 from 11 to 15 years. Fifty known neuroblastoma urine samples from patients attending the Oncology Department and Outpatient Clinic at NHP were also collected for comparison.

Urine samples were collected and divided into two graduated plastic tubes from each participant. The first tube contained $1 \mathrm{ml}$ of $\mathrm{HCl} 6 \mathrm{~mol} / \mathrm{L}$, and urine was added to exactly obtain the total volume of $10 \mathrm{ml}$. Each tube was tested to ensure adequate acidification and the protocol required that any urine samples with a $\mathrm{pH}$ greater than 3.0 were discarded $(n=0)$. The second tube i.e. plain tube was used for creatinine determination. Samples were transported on cold packs to the laboratory within 2 hours of collection. Samples were stored at $-20^{\circ} \mathrm{C}$ until testing and analyzed in batches. Because of adding $\mathrm{HCl}$ for urine HVA and VMA conservation, the final results were obtained by multiplying the first results with a dilution factor.

\section{Measurement of HVA and VMA}

\section{Materials}

HVA, VMA (pure chemicals) were purchased from Sigma (Sigma -Aldrich Chemie GmbH, Riedstrasse 2 D-89555 Steinheim, Germany). Urine Calibrator Lyophylised for VMA/HVA/5-HIAA was obtained from Recipe (Recipe Chemicals + Instruments $\mathrm{GmbH}$, Munich, Germany) and Lyphochek Quantitative Urine Controls were from Bio-Rad (Bio-Rad, Marnes- la- Coquette, France). N,Obis(trimethylsilyl)trifluoroacetamide (BSTFA) was from Supelco (Supelco, Bellfonte, USA). All other solvents and reagents of analytical grade were obtained from Merck (Merck KGaA, Darmstadr, Germany).

\section{Gas chromatography/ mass spectrometry}


An Agilent (Agilent Technologies, Inc. 5301 Stevens Creek Boulevard Santa Clara, CA 95052, USA) Model 7890A gas chromatograph coupled to an Agilent 5975C Mass Selective Detector was used. The gas chromatograph was equipped with a HP-5MS fused- silica capillary column $(30 \mathrm{~m} \times 0.25 \mathrm{~mm}$ id $\times 0.25$ $\mu \mathrm{m}$ film thickness) from Agilent. Helium was used as the carrier gas. The initial column temperature was $160^{\circ} \mathrm{C}$, held for $3 \mathrm{~min}$, followed by an increase at $8^{\circ} \mathrm{C}$ $\mathrm{min}^{-1}$ in first ramp, $50^{\circ} \mathrm{C} \mathrm{m^{-1 }}$ in second ramp held for $3.5 \mathrm{~min}$, to final temperature $280^{\circ} \mathrm{C}$. The total run time was $15.7 \mathrm{~min}$. The transfer line between the GC and MS systems was kept at $290^{\circ} \mathrm{C}$. The ion source temperature was $230^{\circ} \mathrm{C}$.

\section{Sample preparation and derivatization with BSTFA}

A $50 \mu \mathrm{l}$ aliquot of the internal standard solution (containing $5 \mathrm{mM}$ 3-phenyl butyric acid in $20 \mathrm{mM}$ sodium bicarbonate) was added to $1 \mathrm{ml}$ urine sample. This solution was acidified with 2 drops of concentrated $\mathrm{HCl}(37 \%)$, to which a spatula of $\mathrm{NaCl}$ was added to form a saturated solution. After mixing, this solution was extracted with $3 \mathrm{ml}$ of ethyl acetate. The tubes were shaken vigorously for 5 minutes, then centrifuged and the upper layer (organic layer) was carefully removed using a glass pipette. The organic extracts were dried under a stream of nitrogen. $200 \mu \mathrm{l}$ of BSTFA was added and sealed vials were incubated at $80^{\circ} \mathrm{C}$ for 75 minutes. After that the vials were cooled to room temperature and the derivatized samples were transferred onto autosampler vials and $1 \mu \mathrm{l}$ was split injected (split ratio 1:5) onto the GCMS.

\section{HVA, VMA and creatinine quantitation}

The method was calibrated with Recipe Urine Calibrator Lyophylised for VMA/HVA/5-HIAA and internally controlled with Bio-Rad Lyphochek Quantitative urine controls. The target ions were $267 \mathrm{~m} / \mathrm{z}$ for HVA, $371 \mathrm{~m} / \mathrm{z}$ for VMA and 236 $\mathrm{m} / \mathrm{z}$ for internal standard. The qualifier ions were $326 \mathrm{~m} / \mathrm{z}$ for HVA, $297 \mathrm{~m} / \mathrm{z}$ for VMA and $221 \mathrm{~m} / z$ for the internal standard. Formal method validation studies were conducted prior to method implementation. To confirm comparability of 
results with other laboratories in the Australasian region, we participated in the RCPAQAP Urine Chemistry external quality assurance program.

Urine creatinine was measured as a mechanism to correct for concentration. Urine creatinine was measured by Jaffe kinetic method on Beckman Coulter AU2700 chemistry analyser (Beckman Coulter, Mishima, Japan). Patient results were reported relative to creatinine concentration.

\section{Analytical method validation}

Calibration graphs for HVA and VMA were established by using three level standards. The calibration curve was updated each time to minimize the variable conditions. The calibration curve was plotted and the results of the quality control and samples were computed. The method validation studies included linearity, sensitivity, imprecision and recovery experiments.

Limit of Qantitation: The limit of quantification was determined by analyzing serial diluted standards. The diluted standards were assayed five times in a single run according to the standard operation procedures. ${ }^{4}$ Linearity: Two pooled urine samples from children were prepared. One (from healthy children) close to the detection limit and the other (from neuroblastoma patients) near the expected upper limit of the dynamic range. The five mixtures were prepared by mixing the low pool and the high pool with different ratios. Three replicate measurements for each specimen were done. The mean of the measured values and the assigned values were plotted on the linearity graph and inspected for a linear relationship.[5]

Imprecision: The within - batch imprecision was determined by assaying two levels of urine quality control in a single batch $(n=15)$. The betweenbatch imprecision was estimated by analyzing two levels of urine quality control $(n=20)$ over a period of 3 months.

Recovery: For the recovery study, pooled urine from healthy individuals was divided into two aliquots. One aliquot was spiked with the standard solution. The volume of standard added to the sample was less than $10 \%$ 
of that of the pooled urine so that the major disruption of the sample matrix was avoided. The other aliquot was spiked with $0.2 \mathrm{~m} / \mathrm{L} \mathrm{HCl}$ (the solvent for preparing standards). The two aliquots were then analyzed. Five measurements on each aliquot reduced the effects of the imprecision of method.

\section{Ethics Approval}

Ethical approval for collection and analysis of urine samples was obtained from the Ethics Committee at the National Hospital of Pediatrics, Hanoi Vietnam. Informed consent was obtained for all study participants.

\section{Statistical analysis and development of reference intervals:}

One-way analysis of variance (ANOVA) was used to determine if sub-grouped reference intervals were required for gender and age. Due to non-normality, 95\% reference intervals for urinary HVA and VMA were estimated from the sample's 2.5th and 97.5th percentile. Nonparametric bootstrapping methods were employed to generate $95 \%$ confidence intervals around sample reference intervals. 
RESULTS

A urine HVA and VMA GCMS method was developed to diagnose and monitor Neuroblastoma. The chromatography and MS ions monitored in a male urine sample are displayed in Figure 1.

\section{Analytical method validation}

Prior to analysis of the study urine samples, the method was fully validated using protocols from Westgard's Method Validation Textbook.[5] The results of the method validation studies demonstrated that the assay was fit for its clinical purpose. Specific studies conducted were:

\section{Limit of quantitation}

The limit of quantitation for both analytes was $0.9 \mu \mathrm{mol} / \mathrm{L}$.

\section{Linearity}

HVA and VMA were linear to at least 193 and $221 \mu \mathrm{mol} / \mathrm{L}$, respectively. The linear regression for HVA was $y=x-0.1031$. The linear regression for VMA was $y=0.9974 x+0.1951$.

\section{Imprecision}

Using Bio-Rad Lyphochek Quantitative control urine ( $n=15$ at each level), the within batch coefficients of variation (CVs) were, respectively, $2.45 \%$ at $11 \mu \mathrm{mol} / \mathrm{L}$ and $1.20 \%$ at $88 \mu \mathrm{mol} / \mathrm{L}$ for HVA, $2.97 \%$ at $18.2 \mu \mathrm{mol} / \mathrm{L}$ and $1.79 \%$ at $90.6 \mu \mathrm{mol} / \mathrm{L}$ for VMA. The between batch $\mathrm{CVs}$, calculated over 20 consecutive procedures in a 3 month period were $3.6 \%$ at $11 \mu \mathrm{mol} / \mathrm{L}$ and $2.1 \%$ at $88 \mu \mathrm{mol} / \mathrm{L}$ for HVA, $6.6 \%$ at $18.2 \mu \mathrm{mol} / \mathrm{L}$ and $2.6 \%$ at 90.6 $\mu \mathrm{mol} / \mathrm{L}$ for VMA.

\section{Recovery}

Two urines spiked with aqueous standard were used to determine recovery. Recovery for HVA at $38.8 \mu \mathrm{mol} / \mathrm{L}$ and VMA at $27.2 \mu \mathrm{mol} / \mathrm{L}$ were $96 \%$ and $92 \%$ respectively. 


\section{HVA and VMA reference intervals}

The study design collected a total of 684 reference samples from five age groups and 50 samples from neuroblastoma patients (Table 1).

Prior to estimating reference intervals (RIs), the differences between age band and gender means were assessed to determine if Rls were required to be subgrouped for HVA/Creatinine and VMA/Creatinine. The initial step was to fit a twoway factorial analysis of variance (ANOVA) model predicting HVA/Creatinine and VMA/Creatinine using both age bands and gender. However, the Levene's test of homogeneity of variance for these models indicated heteroscedasticity for both HVA/Creatinine, $F(9,624)=18.90, p<.001$ and VMA/Creatinine, $F(9,624)=$ $10.57, p<.001$.

Transformations were explored to correct for heteroscedasticity in the models, but no satisfactory solutions were found. Therefore, the next strategy was to apply a robust Brown-Forsythe method to test the main effects for age bands using one-way ANOVA. The results of these main effect tests were statistically significant for both HVA/Creatinine, $F(4,256.98)=214.13, p<.001$, and $\mathrm{VMA} /$ Creatinine, $F(4,315.20)=97.11, p<.001$. Follow-up pairwise comparisons for using Dunnett's T3 correction for unequal variance revealed all age bands for HVA/Creatinine differed significantly from each other $(p<.001)$. This suggested that all age bands should remain in their sub-groups for $\mathrm{RI}$ estimation.

To determine if age bands should be further divided by gender, individual twosample tests were used to compare means between males and females for each age category. A Bonferroni corrected significance level of $\alpha=0.05 / 5=0.01$ was used to control for inflated type I error across these tests. The results of the twosample $t$-tests found no significant differences between genders on mean HVA/Creatinine across the five age bands (all p-values $>.01$ ). These results suggested that the reference intervals did not need to be sub-divided by gender. Hence the male and female samples per age group were combined (Figure 2a).

The same process was repeated for the statistically significant Brown-Forsythe corrected one-way ANOVA for VMA/Creatinine. Dunnett's T3 multiple comparisons found that all age bands differed significantly from each other 
except for " $<6$ months" vs. " 6 months $<1$ year", $p=.227$ and " $6-10.9$ years" vs. "11 - 15 years", $p=0.017$ (Compared to a Bonferroni corrected significance level of 0.01 ). These results suggested that these age bands could be combined, however, to be consistent with HVA/Creatinine, all five age bands were retained. The two-sample t-tests comparing means between genders across age bands were all not statistically significant. Gender sub-groups were not required for VMA/Creatinine.(Figure 2b)

Reference intervals for HVA/Creatinine and VMA/Creatinine across the five age bands, $<6$ Months, 6 Months $<1,1-5$ Years, 6 - 10 Years, $11-15$ are reported in Table 2. As a comparison, intervals for known Neuroblastoma patients were also calculated for comparison. Reference intervals $(R I)$ were defined as the points where $95 \%$ of measurements will fall such that $2.5 \%$ fall below or above the lower and upper cut-offs respectively. The cut-offs would correspond to estimates of the 2.5 and 97.5 percentile of the population. Due to non-normality, 95\% Bootstrapped [6] confidence intervals for the lower and upper values of the references intervals were calculated (Table 2). 


\section{DISCUSSION}

In this study we successfully developed the urinary HVA - VMA GCMS method based on a classical organic acid profile using a cheaper (non-isotopic) internal standard. We proved that high selectivity and sensitivity of gas chromatography/ mass spectrometry make this technique very suitable for the determination of HVA and VMA in a complex matrix such in urine. The advantages of the method include its fast run time (15.7 minutes), noninvasive sample collection (of random urine), small sample volume (1000 $\mu \mathrm{L}$ of urine), simple sample preparation and cost-effectiveness. In addition, the method is accurate and precise with the added advantage of being cheap. The linearity of the method covers the decision levels of urinary HVA and VMA in diagnosing neuroblastoma. This is a superior alternative to the routine method used before in our laboratory; quantitation of VMA by colorimetric analysis.

The traditional methods for analysis of HVA and VMA include spectrophotometric techniques and gas and liquid chromatography. Unfortunately, the spectrophotometric methods have poor specificity and for this reason, have been replaced by chromatography methods. Liquid chromatography - based methods are the most frequently used.[7] Many of these chromatographic methods employ fluorescence, electrochemistry or mass spectrometry detection to permit simultaneous measurement of both HVA and VMA.[8] GC-MS methods are also quite sensitive and specific and can detect both HVA and VMA simultaneously [8].

Conventionally twenty four hour (24h) urine samples were used for analysis, whilst some studies reported using random urine. Obtaining a complete collection, especially in young infants, is difficult to obtain, time consuming, inconvenient for patients and staff, and lead to higher cost. In addition, children presenting with a suspected neuroblastoma, need rapid investigation and treatment. Since neuroblastoma is the most frequent solid tumor in childhood and represents up to $50 \%$ solid tumors in the first year of life, a reliable but rapid diagnostic strategy to diagnose neuroblastoma in such patients is needed.[9] Thus the age related reference interval of HVA and VMA were established using random urine samples and GCMS method developed in this study. We used 
random urine because of the difficulties of collecting $24 \mathrm{~h}$ urine samples from small children. Random urine samples can be collected easily and there is evidence that this is a valid alternative to timed or $24 \mathrm{~h}$ urine collections.[10]

When using random urine samples to determine reference intervals for HVA and VMA, it is essential to adjust for difference in urinary dilution. Creatinine, which is excreted in the urine at a roughly constant rate, can be used to help make this adjustment. In this study, the reference intervals were constructed based upon the ratios HVA/Creatinine and VMA/Creatinine. The reference data (Table 2) demonstrate that there is a higher excretion of HVA and VMA relative to creatinine early in infancy. The importance of establishing reliable population specific reference intervals for HVA and VMA is highlighted by the possible improvement in prognosis for neuroblastoma with early detection.

Age specific reference intervals for HVA/creatinine and VMA/creatinine have been previously established in other pediatric populations. Some of these studies employed GC without MS detection whilst others used HPLC with various detection systems. Age stratification also varied between these studies.

Some previously studies on other populations have demonstrated similar reference intervals and age stratification to our study. Tuchman et. al. using capillary GC reported random urinary HVA and VMA reference intervals for seven age groups with very low numbers of subjects in each age group.[10] Our values of HVA and VMA for children less than 1 year old were higher but for other groups our results were similar to those of Tuchman.[10] Kellie et al. also used untimed urine samples for HVA and VMA determination [9]. The upper limit of HVA for children less than one year old was also lower than our value, but the value of other age groups were similar. The upper limit of VMA for all age groups was higher than that of ours. Both studies established reference intervals for random urine HVA and VMA using non-neuroblastoma pediatric patients, with all groups greater than one year old mirroring our reference interval data. Fitzgibbon et. al. previously reported the reference values for urinary HVA and VMA in healthy children.[11] The method used was HPLC with electrochemical detection. Henderson et. al. using the capillary GC with flame ionization 
detection published the reference ranges for HVA and VMA excretion in nonneurobastoma pediatric patients.[12] The upper limits of HVA and VMA in these studies are similar to our values. Together, these studies potentially indicate that the reference intervals for HVA and VMA are consistent across ethnic cohorts greater than one year of age.

A number of previous reference interval studies indicate different reference intervals to the ones we report here. Soldin et. al. using HPLC method published HVA and VMA reference values on random urine samples from birth to 20 years.[13] Their upper limit for children of all age groups are lower than that of ours. The method that these authors used was different to our method, which may explain the difference seen between the studies. Recently, Davidson et al. reported the observed ranges for urinary catecholamine and metabolites in random urine specimens from children.[14] They used HPLC with electrochemical detection for measuring HVA and VMA and the age grouping was also different to our study. The upper limit of HVA for children less than 1 year was similar to ours but the upper limit of VMA for all age groups seems to higher than that of ours. Together these studies highlight the caution required when differences in methodology and lack of uniformity in age grouping exists. Our study is the first to look at reference intervals in association with a mass selective detection method.

The purpose of this study was to establish age-specific reference intervals of HVA and VMA in random urines for Vietnamese children. Urinary random HVA and VMA in 50 patients with neuroblastoma are compared with reference intervals. Urinary excretion of HVA exceeded the highest reference value in 45 diagnosed patients $(90 \%)$ and VMA exceeded the highest reference value in 41 diagnosed patients (82\%). Several authors reported that HVA is more consistently elevated in neuroblastoma and that $20 \%$ to $30 \%$ of patients with neuroblastoma excrete normal amounts of VMA.[9] The table 2 indicated there was an overlap between the excretion of HVA and VMA by unaffected children and children with known neuroblastoma on treatment. It has been reported that $10 \%-15 \%$ of histologically proven cases of neuroblastoma are not associated with any increase in excretion of these metabolites.[15,16] Whilst the exact 
upper limit of the reference interval is now defined for random urine HVA and VMA per mmol creatinine due to this lack of sensitivity, clearly elevated values of HVA and VMA are diagnostic but borderline or normal results cannot be used to exclude neuroblastoma. However, the appropriate assignment of the upper reference interval for these biomarkers will ensure maximum specificity.

\section{Conclusion}

A robust testing method for the simultaneous quantitation of urinary HVA and VMA by GCMS was developed. This method is accurate, precise and could be suitable in developing countries where the stable isotope is too expensive. The age - related reference intervals of urinary HVA and VMA were established for Vietnamese children and the values declined progressively with increasing age for each analyte.

\section{Acknowledgements}

This study was supported in part by grants from National Hospital of Pediatrics of Vietnam and Asian-Pacific Federation for Clinical Biochemistry and Laboratory Medicine (APFCB).

We are also grateful to the Prince of the Wales Hospital Hong Kong for technical support, volunteers from preschools and schools in Hanoi, and to the neuroblastoma patients from the Outpatient Clinic and Oncology Department at National Hospital of Pediatrics, Hanoi, Vietnam. 


\section{Tables}

Table 1. Reference Interval Study Groups consisting of 634 normative and 50 know neuroblastoma patients.

\begin{tabular}{|c|c|c|c|c|c|c|c|}
\hline \multirow[b]{3}{*}{ Groups } & \multicolumn{3}{|c|}{ Age } & \multicolumn{4}{|c|}{ Gender } \\
\hline & \multirow[b]{2}{*}{$N$} & \multirow[b]{2}{*}{$M$} & \multirow[b]{2}{*}{$S D$} & \multicolumn{2}{|c|}{ Male } & \multicolumn{2}{|c|}{ Female } \\
\hline & & & & Count & $\%$ & Count & $\%$ \\
\hline$<6$ months & 91 & 0.27 & 0.13 & 35 & $38.5 \%$ & 56 & $61.5 \%$ \\
\hline 6 months $<1$ year & 141 & 0.71 & 0.15 & 59 & $41.8 \%$ & 82 & $58.2 \%$ \\
\hline 1 - 5.9 years & 139 & 3.62 & 1.22 & 66 & $47.5 \%$ & 73 & $52.5 \%$ \\
\hline $6-10.9$ years & 136 & 8.30 & 1.23 & 64 & $47.1 \%$ & 72 & $52.9 \%$ \\
\hline $11-15$ years & 127 & 13.26 & 1.24 & 72 & $56.7 \%$ & 55 & $43.3 \%$ \\
\hline Neuroblastoma & 50 & 3.46 & 1.91 & 22 & $44.0 \%$ & 28 & $56.0 \%$ \\
\hline
\end{tabular}


Table 2. Reference Interval Estimates and 95\% Bootstrapped Confidence Intervals for HVA/Creatinine and VMA/Creatinine

\begin{tabular}{|c|c|c|c|c|c|c|c|}
\hline \multirow[b]{2}{*}{ Outcome } & \multirow[b]{2}{*}{ Age Band } & \multirow[b]{2}{*}{$N$} & \multirow[b]{2}{*}{ Mean } & \multirow[b]{2}{*}{$S D$} & \multirow[b]{2}{*}{ Median } & \multicolumn{2}{|c|}{ Reference Interval Estimates ${ }^{a}$} \\
\hline & & & & & & Lower & Upper \\
\hline \multirow[t]{6}{*}{ HVA/Creatinine } & $<6$ Months & 91 & 17.70 & 6.99 & 17.08 & $\begin{array}{c}6.17 \\
(3.90 .8 .58)\end{array}$ & $\begin{array}{c}36.1 \\
(27.43 .3966)\end{array}$ \\
\hline & 6 Months $<1$ & 141 & 13.24 & 5.30 & 12.96 & $\begin{array}{c}3.74 \\
(1.83,5.06)\end{array}$ & $\begin{array}{c}25.91 \\
(20.86,31.62)\end{array}$ \\
\hline & 1 - 5 Years & 139 & 6.85 & 3.29 & 5.96 & $\begin{array}{c}3.50 \\
(2.94,4.09)\end{array}$ & $\begin{array}{c}16.5 \\
(12.85,20.79)\end{array}$ \\
\hline & $6-10$ Years & 136 & 4.54 & 1.79 & 4.14 & $\begin{array}{c}2.73 \\
(2.37,2.86)\end{array}$ & $\begin{array}{c}8.25 \\
(7.00,9.73)\end{array}$ \\
\hline & 11 - 15 Year & 127 & 3.42 & 1.96 & 2.92 & $\begin{array}{c}1.24 \\
(0.96,1.63)\end{array}$ & $\begin{array}{c}9.03 \\
(6.28,14.18)\end{array}$ \\
\hline & Neuroblastoma & 50 & 126.87 & 143.33 & 71.05 & $\begin{array}{c}5.56 \\
(3.80,15.0)\end{array}$ & $\begin{array}{c}510.14 \\
(337.1,579.1)\end{array}$ \\
\hline \multirow[t]{6}{*}{ VMA/Creatinine } & $<6$ Months & 91 & 6.10 & 2.69 & 5.60 & $\begin{array}{c}2.04 \\
(1.74,2.80)\end{array}$ & $\begin{array}{c}11.94 \\
(10.70,16.48)\end{array}$ \\
\hline & 6 Months < 1 & 141 & 5.38 & 1.83 & 5.36 & $\begin{array}{c}1.65 \\
(0.26,2.64)\end{array}$ & $\begin{array}{c}9.05 \\
(8.13,9.73)\end{array}$ \\
\hline & 1 - 5 Years & 139 & 3.62 & 1.27 & 3.28 & $\begin{array}{c}2.09 \\
(1.55,2.36)\end{array}$ & $\begin{array}{c}7.36 \\
(5.91,8.57)\end{array}$ \\
\hline & 6 - 10 Years & 136 & 2.82 & 1.06 & 2.66 & $\begin{array}{c}1.70 \\
(1.45,1.87)\end{array}$ & $\begin{array}{c}4.36 \\
(3.88,6.49)\end{array}$ \\
\hline & $11-15$ Year & 127 & 2.31 & 1.51 & 2.05 & $\begin{array}{c}0.02 \\
(0.01,0.20)\end{array}$ & $\begin{array}{c}5.62 \\
(4.05,6.99)\end{array}$ \\
\hline & Neuroblastoma & 50 & 83.06 & 103.63 & 46.55 & $\begin{array}{c}2.34 \\
(1.70,4.87)\end{array}$ & $\begin{array}{c}313.59 \\
(180.1,560.8)\end{array}$ \\
\hline
\end{tabular}


Figures Legend

Figure 1a: Total lon Chromatogram (TIC) of the urine sample taken from a neuroblastoma patient

Figure 1b. Electron Impact (EI) mass spectra of HVA

Figure 1c. Electron impact (EI) mass spectra of VMA

Figure $2 \mathrm{a}$ - Box plots for HVA/Creatinine (umol/mmol) across age bands. Circles represent outliers and stars represent extreme outliers. Outliers give all age band distributions a positive skew. Variance decreases with age.

Figure $2 \mathrm{~b}$ - Box whisker plots VMA/creatinine (umol $/ \mathrm{mmol}$ ) across age bands. Circles represent outliers and stars represent extreme outliers. Outliers give all age band distributions a positive skew. Variance decreases with age. 
1 Fauler G, Leis H.J, Huber E, Schellauf Ch, Urban Ch., Gleispach H. Determination of homovanillic acid and vanillylmandelic acid in neuroblastoma Screening by stable isotope dilution GC-MS. Journal of Mass Spectrometry 1997; 32: 507- 514.

2 Tuchman M, Ramnaraine ML, Woods WG, Krivit W. Three years experience with random urinary homovanillic and vanillylmandelic acid levels in the diagnosis of neuroblastoma. Pediatrics 1987, 79: 203- 205.

3 Boneh A. Greaves R. Garra G. Casanelia S. Warwick L. Pitt J. Metabolic management of pregnancy and post - delivery period in a patient with Cobalamin A disease. American Journal of Obstetrics and Gynaecology. 2002;187:225-6.

4 Armbruster DA, Pry T. Limit of blank, limit of detection and limit of quantitation. Clin Biochem Rev. 2008;29:S49-S52.

5 Westgard, J.O. 1999. Basic Method Validation. Westgard Quality Corporation. pp250.

6 Efron B. Bootstrap methods: Another look at the jackknife. The Annals of Statistics, 1979; 7: 1-26.

7 RCPAQAP Urine Chemistry and Biogenic Amines Program. End of cycle report for cycle 55, 2012. www.rcpaqap.com.au/chempath Accessed 15th July 2013.

8 Allenbrand R and Garg U. Quantitation of Homovanillic Acid and Vanillylmandelic Acid in urine using Gas Chromatography- Mass Spectrometry. Clinical Applications of Mass Spectrometry, Methods in molecular Biology 2010; 261- 269.

9 Kellie S.J, Clague A.E, H.M McGeary, and P.J.Smith. The value of catecholamine metabolite determination on untimed urine collections in the diagnosis of neural crest tumours in children. Aust.Paediatr. J 1986; 22: 313-315.

10 Tuchman M, Christopher L. Morris, Margaret L. Ramnaraine, Lary D. Bowers and William Krivit. Value of Random Urinary Homovanillic acid and Vanillylmandelic acid levels in the Diagnosis and Management of Patients with Neuroblastoma: Comparison with 24-Hour urine collections. Pediatrics 1985; 75(2): 324- 328.

11 Fitzgibbon M, FitzGerald RJ, Tormey WP, O’MearaA, Kenny D. Reference values for 
urinary HMMA, HVA, noradrenaline, adrenaline, and dopamine excretion in children using random urine samples and HPLC with electrochemical detection. Ann Clin Biochem 1992; 29(4): 400- 404.

12 Henderson M J, Heney D, McGinlay J M, Lewis I and Bailey C. Measurement of dopamine, HVA and HMMA in untimed urine samples: establishment of age- related reference data in children. Ann Clin Biochem 1992; 29: 162- 167.

13 Soldin SJ, Lam G, Pollard, Allen LC, Logan AG. High-performance liquid chromatographic analysis of urinary catecholamines employing amperometric detection: reference values and use in laboratory diagnosis of neural crest tumours. Clin Biochem 1980; 13: 285-291.

14 Davison DF, Hammond PJ, Murphy D, Carachi R. Age-related medical decision limits for urinary free (unconjugated) metadrenalines, catecholamines and metabolites in random urine specimens from children. Annals of Clinical Biochemistry 2011; 48(4): 358-66.

15 LaBrosse EH, Comoy E, Bohuon C, et al. Catecholamine metabolism in neuroblastoma. J Natl Cancer Inst, 1976; 57: 633- 638.

16 Pritchard J, Barnes J, Germond S et al. Stage and urinary catecholamine metabolite excretion in neuroblastoma. Lancet 1989; ii: 514- 515. 


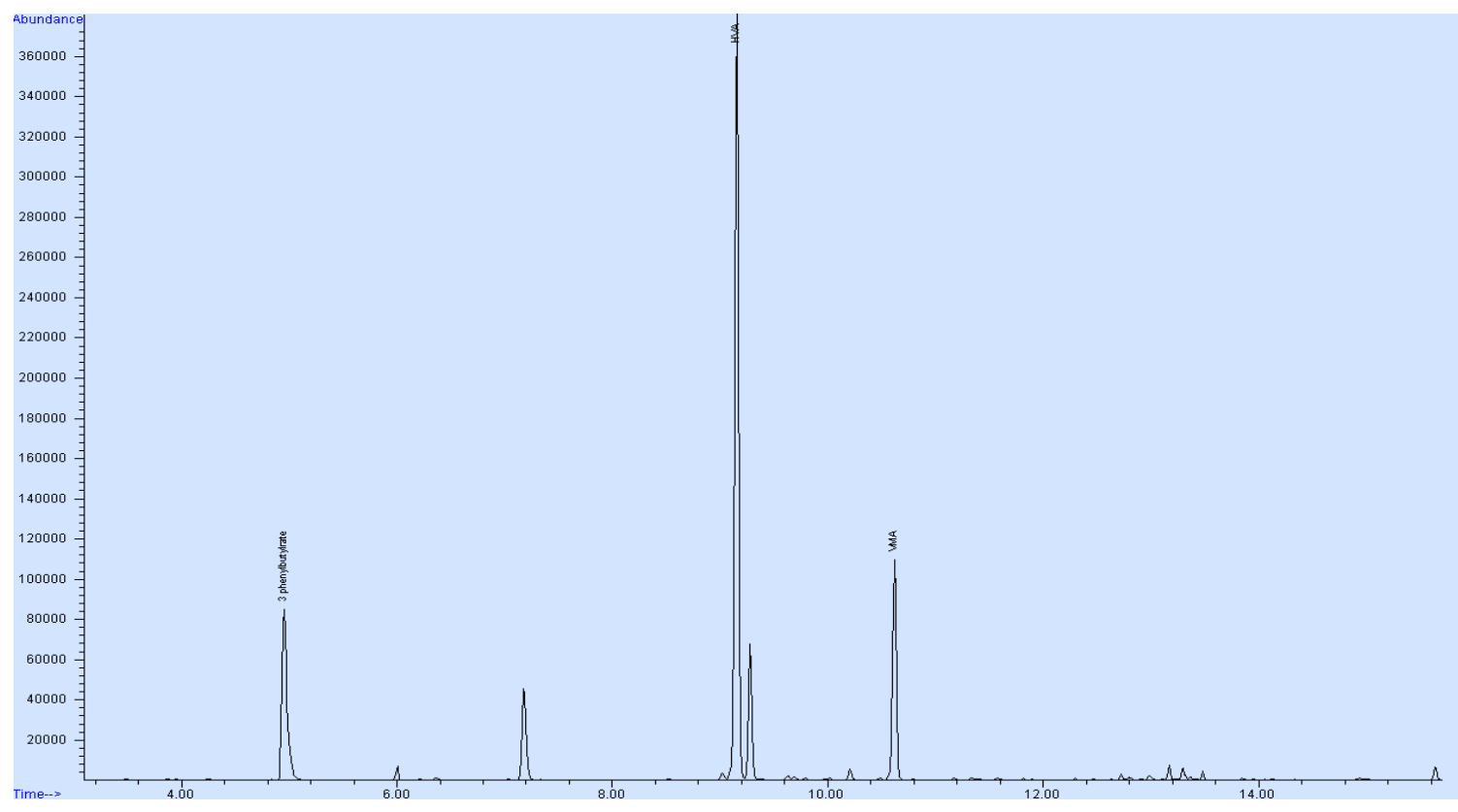

Figure 1a: Total lon Chromatogram (TIC) of the urine sample taken from a neuroblastoma patient

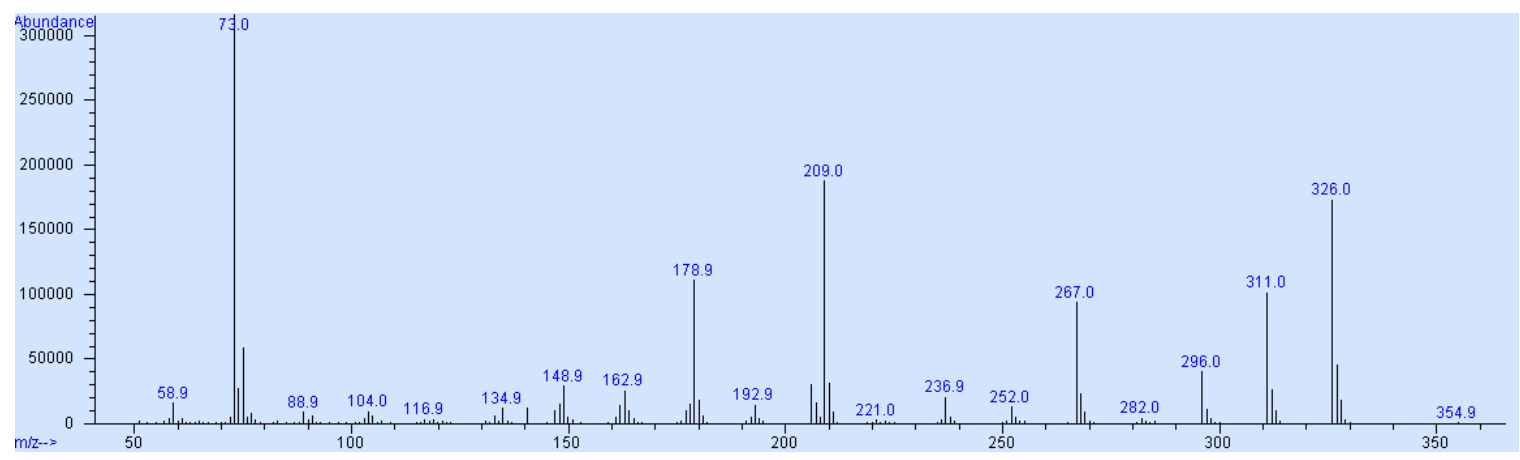

Figure 1b. Electron Impact (EI) mass spectra of HVA

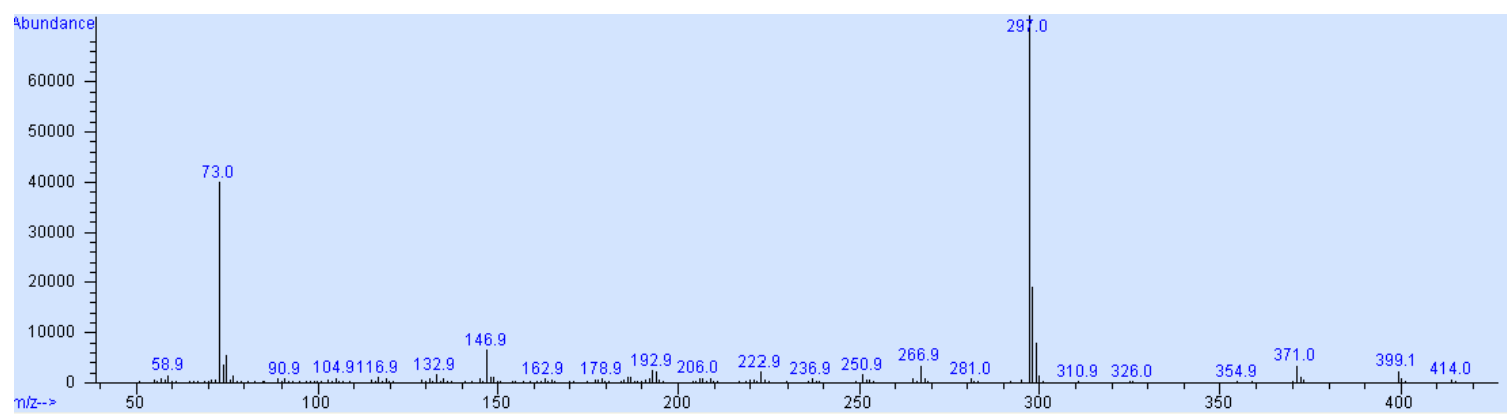

Figure 1c. Electron impact (EI) mass spectra of VMA 


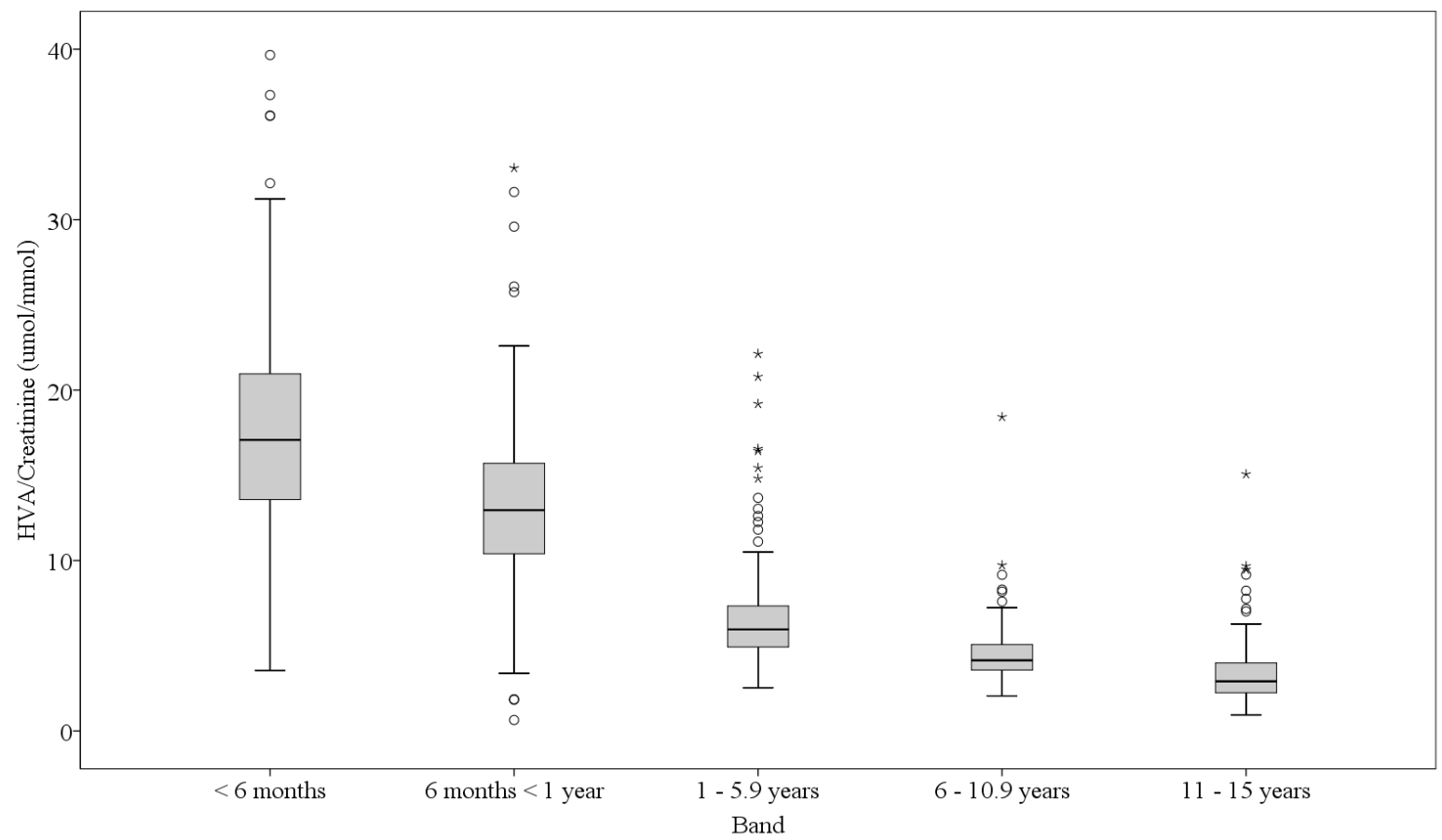

Figure $2 \mathrm{a}$ - Box plots for HVA/Creatinine (umol $/ \mathrm{mmol}$ ) across age bands. Circles represent outliers and stars represent extreme outliers. Outliers give all age band distributions a positive skew. Variance decreases with age.

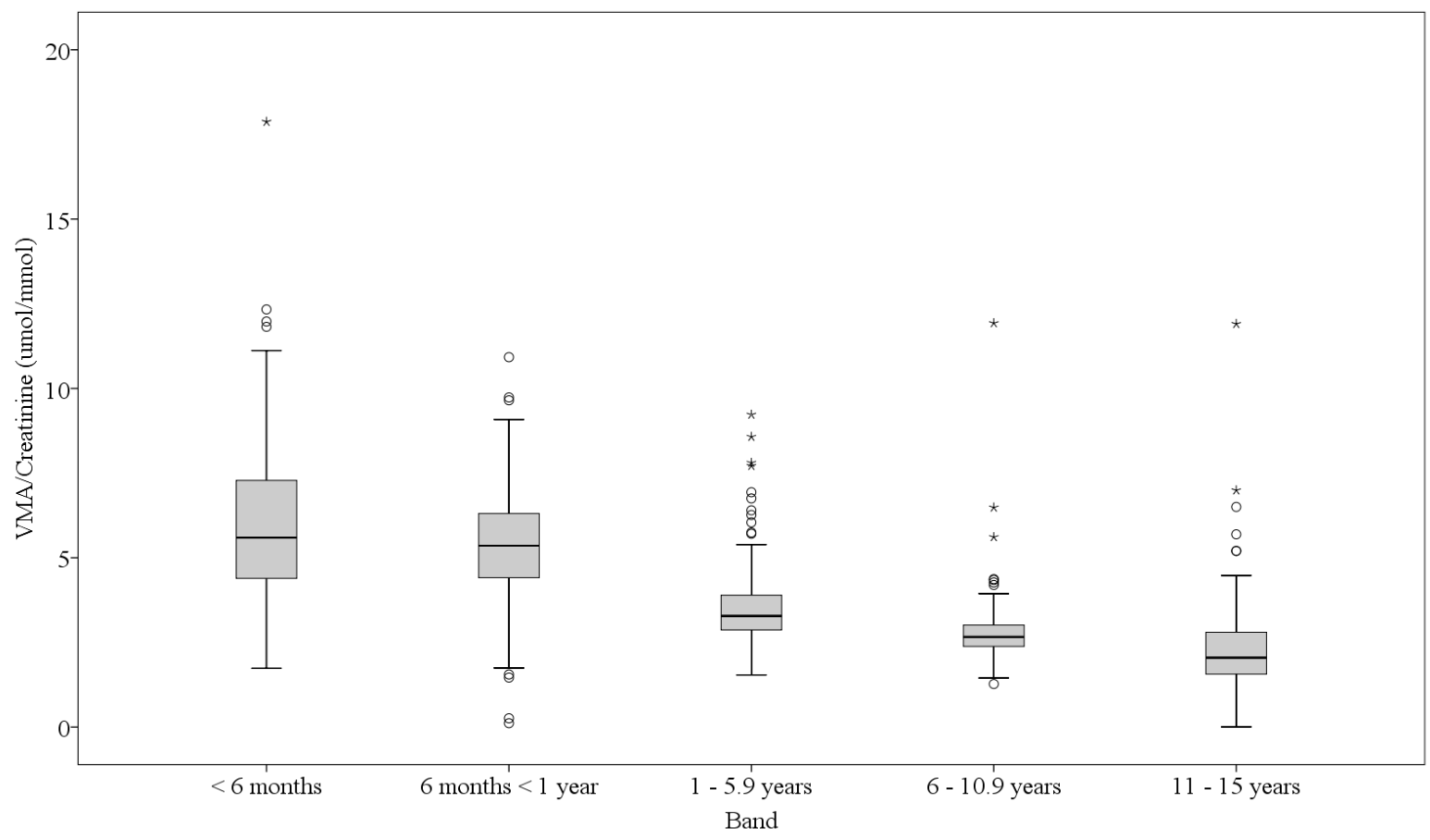

Figure $2 \mathrm{~b}$ - Box whisker plots VMA/creatinine (umol $/ \mathrm{mmol}$ ) across age bands. Circles represent outliers and stars represent extreme outliers. Outliers give all age band distributions a positive skew. Variance decreases with age. 